\title{
A GENERALIZATION OF THEOREMS OF RELLICH AND ATKINSON
}

\author{
CALVIN H. WILCOX
}

The wave functions $u(\boldsymbol{r})$ which describe the scattering of timeharmonic waves (acoustical, electromagnetic, etc.) by bounded scatterers satisfy the Helmholtz equation and Sommerfeld's radiation condition

$$
\lim _{r \rightarrow \infty} r\left(\frac{\partial u}{\partial r}-i k u\right)=0, \quad \text { uniformly in } \theta, \phi .
$$

In deriving the properties of such functions the integral representation

$$
u(\boldsymbol{r})=\frac{1}{4 \pi} \int_{r^{\prime}-r_{0}}\left\{u \frac{\partial}{\partial r^{\prime}}\left(\frac{e^{i k \rho}}{\rho}\right)-\frac{e^{i k_{\rho}}}{\rho} \frac{\partial u}{\partial r^{\prime}}\right\} d S^{\prime}, \quad r>r_{0},
$$

where $\rho=\left|\boldsymbol{r}-\boldsymbol{r}^{\prime}\right|$, plays a fundamental role. In deducing (2) it is usual to introduce additional hypotheses on $u(r)$ (see, e.g. [2, pp. 23-26]), notably Sommerfeld's "condition of finiteness" [6]

$$
u(r)=O(1 / r), \quad r \rightarrow \infty .
$$

In the present paper it is shown how (2) may be derived without such additional assumptions. The utility of (2) is then demonstrated by deriving two important theorems for wave functions from it.

1. A statement of results.

Definition. An exterior region $V$ is a region (open connected set) consisting of all points outside a closed bounded surface $S$.

Definition. A radiation function for the exterior region $V$ is a complex-valued function $u(r)$ which satisfies

A. $u(r)$ is of class $C^{2}$ and satisfies the Helmholtz equation

$$
\nabla^{2} u(\boldsymbol{r})+k^{2} u(\boldsymbol{r})=0
$$

in the closure of $V$, and

B. $u(\boldsymbol{r})$ satisfies Sommerfeld's radiation condition, ${ }^{1}$

$$
\lim _{R \rightarrow \infty} \int_{r=R}\left|\frac{\partial u(\boldsymbol{r})}{\partial r}-i k u(\boldsymbol{r})\right|^{2} d S=0,
$$

Presented to the Society, June 18, 1955; received by the editors May 9, 1955.

1 Sommerfeld's original condition was the somewhat stronger statement (1) above (see [6]). 
where $k \neq 0$ is a complex number satisfying $\operatorname{Im} k \geqq 0 .^{2}$ Here $\boldsymbol{r}$ is a position vector and $(r, \theta, \phi)$ are spherical coordinates for it.

The main result of this paper is that any radiation function may be represented in the form (2). The proof is based on a lemma.

LEMMA. Let $u(\boldsymbol{r})$ be a radiation function and write $\boldsymbol{r}=r \hat{\omega}$ where $\hat{\omega}$ is $a$ unit vector. Then

$$
\int_{\Omega}|u(r \omega)|^{2} d \Omega=O\left(\frac{1}{r^{2}}\right), \quad r \rightarrow \infty
$$

and

$$
\int_{\Omega}\left|\frac{\partial u(r \omega)}{\partial r}\right|^{2} d \Omega=O\left(\frac{1}{r^{2}}\right), \quad r \rightarrow \infty,
$$

where $\Omega$ is the unit sphere and $d \Omega$ is the element of solid angle. Moreover if $\operatorname{Im} k>0$ then the improper integrals

$$
\int_{V}|u(r)|^{2} d V
$$

and

$$
\int_{V}|\nabla u(\mathbf{r})|^{2} d V
$$

are convergent. The surface $S$ of $V$ must be such that the divergence theorem is applicable, e.g. regular in the sense of Kellogg [4, p. 112].

With the help of the lemma it is easy to prove the following

REPRESENTATION THEOREM. Let $u(\boldsymbol{r})$ be a radiation function for an exterior region $V$ bounded internally by a regular surface $S$. Then

$$
u(\boldsymbol{r})=\frac{1}{4 \pi} \int_{S}\left\{u \frac{\partial}{\partial n}\left(\frac{e^{i k \rho}}{\rho}\right)-\frac{e^{i k \rho}}{\rho} \frac{\partial u}{\partial n}\right\} d S^{\prime}
$$

where $\hat{n}$ is a unit normal to $S$, pointing into $V$, and $\rho=\left|\boldsymbol{r}-\boldsymbol{r}^{\prime}\right|$.

From this result one may infer the

EXPANSION THEOREM. Let $u(r)$ be a radiation function for the region exterior to a sphere $\left|\boldsymbol{r}-\boldsymbol{r}_{0}\right|=c$, and let $(r, \theta, \phi)$ be spherical coordinates for $r$ relative to an origin at $r_{0}$. Then

2 This is a natural restriction since, in the applications to physical problems, $k^{2}$ is in the first quadrant and $k$ is the root of $k^{2}$ which is positive when $k^{2}$ is real. 


$$
u(\boldsymbol{r})=\frac{e^{i k r}}{r} \sum_{n=0}^{\infty} \frac{f_{n}(\theta, \phi)}{r^{n}}
$$

where the series converges for $r>c$ and converges absolutely and uniformly in $r, \theta$ and $\phi$ in any region $r \geqq c+\epsilon>c$. The series may be differentiated term by term with respect to $r, \theta$, and $\phi$ any number of times and the resulting series all converge absolutely and uniformly.

CoRollary. The coefficients $f_{n}(\theta, \phi), n>0$, may be determined from the "radiation pattern" $f_{0}(\theta, \phi)$ by the recursion formula

$$
2 i k n f_{n}=n(n-1) f_{n-1}+D f_{n-1}, \quad n=1,2, \cdots,
$$

where

$$
D f=\frac{1}{\sin \theta} \frac{\partial}{\partial \theta}\left(\sin \theta \frac{\partial f}{\partial \theta}\right)+\frac{1}{\sin ^{2} \theta} \frac{\partial^{2} f}{\partial \phi^{2}}
$$

is Beltrami's operator for the sphere. Hence, in particular, $u(\boldsymbol{r})$ is determined in the region $r>c$ by its radiation pattern.

The expansion theorem and its corollary imply the following

UNIQUENESS THEOREM. Let $V$ be an exterior region bounded internally by a regular surface $S$ (in the sense of Kellogg, [4, p. 112]), and let $f$ be an arbitrary continuous function on $S$. Then there is at most one radiation function $u(r)$ for $V$ which satisfies the boundary condition

C.

$$
u=f \quad \text { on } S, \text { or } \frac{\partial u}{\partial n}=f \quad \text { on } S .
$$

Here $\hat{n}$ is a unit normal vector to $S$.

2. A discussion of related literature. Versions of the representation, uniqueness and expansion theorems have been proved, under stronger hypotheses, by Baker and Copson, Rellich, Atkinson, and Barrar and Kay. Rellich [5], in 1943, proved the uniqueness theorem under the additional assumption that the "wave number" $k$ is real and positive. The generalization of the theorem to the domain $\operatorname{Im} k \geqq 0$ is important in the applications to scattering problems where it is often necessary to require Im $k>0$ to secure the convergence of integrals. Baker and Copson [2, pp. 23-26] have proved the representation theorem for radiation functions satisfying the additional "condition of finiteness" of Sommerfeld [6].

D.

$$
u(r \hat{\omega})=O(1 / r),
$$$$
r \rightarrow \infty \text {, uniformly in } \hat{\omega} \text {. }
$$

Atkinson [1], in 1947, proved that radiation functions satisfying 
D could be expanded in a series (4) which converged uniformly and absolutely. This result has recently been refined by Barrar and Kay [3] who showed that, under Atkinson's hypotheses, the series (4) can be differentiated term by term any number of times and that the recursion formula (5) holds. They also sketched a proof that condition $\mathrm{D}$ is not needed, but the author believes that their proposed proof is incorrect.

3. A proof of the lemma. The radiation condition B may be written

$$
\lim _{R \rightarrow \infty} \int_{r=R}\left\{\left|\frac{\partial u}{\partial r}\right|^{2}+|k|^{2}|u|^{2}+i \bar{k} \bar{u} \frac{\partial u}{\partial r}-i k u \frac{\partial \bar{u}}{\partial r}\right\} d S=0 .
$$

Green's first identity $[4$, p. 212]

$$
\int_{R} v \nabla^{2} u d V+\int_{R} \nabla v \cdot \nabla u d V=\int_{\Sigma} v \frac{\partial u}{\partial n} d S
$$

is now applied with $v=\bar{u}$ and $R$ replaced by the region $V_{R}$ between $S$ and the large sphere $r=R$. Since $u$ satisfies $A$ this gives

$$
\begin{aligned}
i \bar{k} \int_{r=R} \bar{u} \frac{\partial u}{\partial r} d S= & i \bar{k} \int_{S} \bar{u} \frac{\partial u}{\partial n} d S-i k|k|^{2} \int_{V_{R}}|u|^{2} d V \\
& +i \bar{k} \int_{V_{R}}|\nabla u|^{2} d V .
\end{aligned}
$$

If the complex conjugate of equation (ii) is added to (ii) and the result put in equation (i) then (i) becomes

$$
\begin{array}{r}
\lim _{R \rightarrow \infty}\left[\int_{r=R}\left|\frac{\partial u}{\partial r}\right|^{2} d S+|k|^{2} \int_{r=R}|u|^{2} d S+2 \operatorname{Im} k \int_{\nabla_{R}}|\nabla u|^{2} d V\right. \\
\left.+2|k|^{2} \operatorname{Im} k \int_{V_{R}}|u|^{2} d V\right]=2 \operatorname{Im}\left\{k \int_{S} u \frac{\partial \bar{u}}{\partial n} d S\right\} .
\end{array}
$$

Now the right member of (iii) is a finite number independent of $R$. Thus, since the four terms in the left hand member are all nonnegative ( $\operatorname{Im} k \geqq 0$ ), the four terms on the left must be individually bounded. Since $d S=R^{2} d \Omega$ on $r=R$ and $|k| \neq 0$ the first two terms yield

$$
\int_{\Omega}\left|\frac{\partial u(r \hat{\omega})}{\partial r}\right|^{2} d \Omega=O\left(\frac{1}{r^{2}}\right), \quad \int_{\Omega}|u(r \hat{\omega})|^{2} d \Omega=O\left(\frac{1}{r^{2}}\right), \quad r \rightarrow \infty .
$$

If Im $k>0$ then the last two integrals on the left are bounded for $R \rightarrow \infty$. Since they are also monotone increasing functions of $R$ this implies that the improper integrals 


$$
\int_{V}|u|^{2} d V \text { and } \int_{V}|\nabla u|^{2} d V
$$

are convergent, which completes the proof.

4. Proofs of the representation, expansion, and uniqueness theorems. Baker and Copson, in their proof of the representation theorem [2, pp. 23-26], show that if $u(r)$ is of class $C^{2}$ and satisfies the Helmholtz equation in $V$ then

$$
u(\boldsymbol{r})=\frac{1}{4 \pi} \int_{S}\left\{u \frac{\partial}{\partial n}\left(\frac{e^{i k \rho}}{\rho}\right)-\frac{e^{i k \rho}}{\rho} \frac{\partial u}{\partial n}\right\} d S
$$

$$
+\frac{1}{4 \pi} \int_{\Omega} e^{i k R} R\left\{\frac{\partial u}{\partial r}-i k u\right\}_{r=R} d \Omega+\frac{1}{4 \pi} \int_{\Omega} e^{i k R}\{u\}_{r=R} d \Omega
$$

where $(r, \theta, \phi)$ are spherical coordinates with origin at $\boldsymbol{r}$. In particular (iv) holds for any radiation function. But $\left|e^{i k R}\right| \leqq 1$ for $\operatorname{Im} k \geqq 0$. Hence Schwarz's inequality yields

$$
\left|\frac{1}{4 \pi} \int_{\Omega} e^{i k R} R\left\{\frac{\partial u}{\partial r}-i k u\right\} d \Omega\right|^{2} \leqq \frac{1}{4 \pi} \int_{r=R}\left|\frac{\partial u}{\partial r}-i k u\right|^{2} d S
$$

and

$$
\left|\frac{1}{4 \pi} \int_{\Omega} e^{i k R} u d \Omega\right|^{2} \leqq \frac{1}{4 \pi} \int_{\Omega}|u|^{2} d \Omega .
$$

The first integral tends to zero by the radiation condition and the second is $O\left(1 / R^{2}\right)$ by the lemma. Thus the last two integrals in (iv) tend to zero with $1 / R$ and the representation theorem follows.

The expansion theorem and its corollary follow directly from the representation theorem by the methods of Atkinson and Barrar and Kay and the proofs are not, therefore, reproduced here.

Turning now to the uniqueness theorem, let $u$ and $v$ be any two radiation functions for $V$ which satisfy $C$. Then $w=u-v$ is a radiation function for $V$ which satisfies $C$ with $f \equiv 0$. This is not immediately apparent since defining condition $B$ for radiation functions is not linear. However, by the expansion theorem both $u$ and $v$, and therefore $w$, have expansions of the form (4) which converge absolutely and uniformly and may be differentiated term by term. It follows easily that $w$ is a radiation function for $V$. Hence equation (iii) holds with the right-hand term equal to zero. In the case Im $k>0$ the remainder of the proof is particularly simple, for then (iii) implies that 


$$
\int_{V}|w|^{2} d V=0
$$

whence $w \equiv 0$ in $V$. If $k$ is real the last two terms on the left in (iii) disappear. However, since $|k| \neq 0$, the second term yields

$$
\lim _{R \rightarrow \infty} \int_{r=R}|w|^{2} d S=0 .
$$

By the expansion theorem

$$
w=\frac{e^{i k r}}{r} \sum_{n=0}^{\infty} \frac{a_{n}}{r^{n}}
$$

where the series converges absolutely and uniformly in $r, \theta$, and $\phi$ outside any sphere $r=r_{0}$ containing $S$. Thus

$$
\int_{r=R}|w|^{2} d S=\int_{\Omega}\left|a_{0}(\hat{\omega})\right|^{2} d \Omega+\frac{1}{R} \int_{\Omega}\left(\bar{a}_{0} a_{1}+a_{0} \bar{a}_{1}\right) d \Omega+\cdots
$$

where the series converges in $R \geqq r_{0}$. Thus making $R \rightarrow \infty$ gives

$$
\int_{\Omega}\left|a_{0}(\hat{\omega})\right|^{2} d \Omega=0
$$

whence, since $a_{0}$ is continuous, $a_{0}(\hat{\omega}) \equiv 0$. But then the recursion formula of the corollary to the expansion theorem implies $a_{n}(\hat{\omega}) \equiv 0$, $n=1,2, \cdots$, and hence $w(r) \equiv 0$ in $r>r_{0}$. But $w(r)$ is analytic in the whole region $V$ (this is an immediate consequence of the representation theorem) and hence must vanish in all of $V$ since it vanishes in a subdomain. This completes the proof of the theorem.

\section{BIBLIOGRAPHY}

1. F. V. Atkinson, On Sommerfeld's "radiation condition," The Philosophical Magazine vol. 40 (1949) pp. 645-651.

2. B. B. Baker and E. T. Copson, The mathematical theory of Huygens' principle, 2d ed., Oxford, 1950.

3. R. B. Barrar and A. F. Kay, A series development of a solution of the wave equation in powers of $1 / r$, Technical Research Group, New York City, un published.

4. O. D. Kellogg, Foundations of potential theory, New York, Ungar, 1929.

5. F. Rellich, Über das asymptotische Verhalten der Lösungen von $\Delta u+k u=0$ im unendlichen Gebieten, Jber. Deutschen Math. Verein. vol. 53 (1943) pp. 57-64.

6. A. Sommerfeld, Die Greensche Funktion der Schwingungsgleichung, Jber. Deutschen Math. Verein. vol. 21 (1912) pp. 309-353.

HARVARD UNIVERSITY 